\title{
A Lattice Boltzmann Method for a Binary Miscible Fluid Mixture and Its Application to a Heat Transfer Problem
}

\author{
Takaji Inamuro $^{\dagger}$, Masato Yoshino ${ }^{1}$, Hiroshi Inoue ${ }^{2}$, Riki Mizuno $^{3}$, and Fumimaru Ogino \\ Department of Chemical Engineering, Graduate School of Engineering, Kyoto University, \\ Kyoto 606-8501, Japan
}

\footnotetext{
${ }^{\dagger}$ Corresponding author,

Address: Yoshida-honmachi, Sakyo-ku, Kyoto 606-8501, Japan

E-mail: inamuro@cheme.kyoto-u.ac.jp

Tel: $+81-75-753-5581$

Fax: +81-75-761-7695

${ }^{1}$ Present address: Department of Mechanical Systems Engineering, Faculty of Engineering, Shinshu University, Nagano 380-8553, Japan; E-mail: masato@gipwc.shinshu-u.ac.jp

${ }^{2}$ Present address: Canon Inc., Ota-ku, Tokyo 146-8501, Japan; E-mail: inoue.hiroshi@canon.co.jp

${ }^{3}$ Present address: NEC Corporation, Sagamihara, Kanagawa 229-1198, Japan;

E-mail: r-mizuno@cq.jp.nec.com
}

D R A F T February 15, 2002, 1:27pm D R A F T 
A lattice Boltzmann method (LBM) for an isothermal binary miscible fluid mixture is proposed. The binary miscible fluid mixture is assumed to be composed of $A$ - and $B$-species where the fraction of $B$-species is much smaller than that of $A$-species. The asymptotic theory proposed by Sone [in Rarefied Gas Dynamics, edited by D. Dini (Editrice Tecnico Scientifica, Pisa, 1971), Vol. 2, p. 737] is applied to the present LBM model and the convection-diffusion equation for component $B$ is obtained. A diffusion problem is calculated and the validity of the proposed model is shown. Also, the present method can be applied to thermal fluid systems, in which the concentration field of component $B$ is regarded as the temperature field of component $A$ and a buoyancy force proportional to the temperature difference is included. Rayleigh-Bénard convection is numerically simulated. The results indicate that the present LBM is useful for the simulation of fluid flows with heat transfer as well as mass transfer.

Key Words: lattice Boltzmann method (LBM); binary miscible fluid mixture; asymptotic analysis; Rayleigh-Bénard convection; heat transfer; mass transfer

Mathematical Reviews Index:

65C20 Models, numerical methods,

80A20 Heat and mass transfer, heat flow

\section{R A F T February 15, 2002, 1:27pm D R A F T}




\section{INTRODUCTION}

Recently, the lattice Boltzmann method (LBM) [1-4] has been used for many kinds of simulations of incompressible viscous flows. In particular, LBM has been successfully applied to problems of multiphase and multicomponent fluid flows [510]. Gunstensen et al. [5] developed a multicomponent LBM based on the twocomponent lattice gas model proposed by Rothman and Keller [6]. Holme and Rothman [7] extended the method to reduce the diffusivity in a miscible two-component system. Flekkøy [8] introduced another two-component LBM model composed of two miscible fluids. Shan and Doolen $[9,10]$ proposed a multicomponent LBM model including interparticle interaction and external forces.

On the other hand, LBM has also been applied to problems of fluid flows including thermal effects. In general, however, the simulation of thermal fluid systems by LBM has not achieved the same success as that of isothermal flows. For example, McNamara et al. [11] developed a three-dimensional multispeed thermal LBM. While their method gives accurate results, they pointed out that the numerical stability should be improved. Most previous thermal LBM models [11-14] are based on such a multispeed approach in which additional particle speeds are needed to obtain the energy equation at the macroscopic level.

As an alternative approach, Shan [15] carried out numerical simulations of RayleighBénard convection by using the previously developed two-component LBM. In his method, the density field of the second component is used to simulate the temperature field by taking advantage of the formal analogy between heat and mass transfer. He et al. [16] also developed a novel thermal LBM model based on a similar approach, in which an independent distribution function for internal energy is introduced to simulate the temperature field. These models are numerically more stable than that of the multispeed thermal LBMs. In both models, however, the equilibrium distribution function for the temperature contains the terms of second

\section{R A F T February 15, 2002, 1:27pm D R A F T}


order in flow velocity. Since the convection-diffusion equation for the temperature has no terms of second order in flow velocity, it is sufficient to take account of the terms up to first order in flow velocity [17]. Thus there is a possibility of simplifying their models.

In the present paper, we first propose an LBM for an isothermal binary miscible fluid mixture. Next we apply the asymptotic theory proposed by Sone [18-21] to the present LBM model and obtain the convection-diffusion equation for the diffusing component. Then we calculate a diffusion problem to demonstrate the validity of the proposed method. Finally we apply the present LBM to thermal fluid systems and carry out numerical simulations of Rayleigh-Bénard convection.

\section{LATTICE BOLTZMANN METHOD FOR BINARY MISCIBLE \\ FLUID MIXTURE}

\subsection{Basic Equation}

Hereafter, non-dimensional variables defined in Appendix A are used, but the circumflex representing 'non-dimensional' is omitted for simplicity. We employ the fifteen-velocity model [4] to explain the present method. The fifteen-velocity model has the following velocity vectors:

$$
\begin{aligned}
& {\left[\boldsymbol{c}_{1}, \boldsymbol{c}_{2}, \boldsymbol{c}_{3}, \boldsymbol{c}_{4}, \boldsymbol{c}_{5}, \boldsymbol{c}_{6}, \boldsymbol{c}_{7}, \boldsymbol{c}_{8}, \boldsymbol{c}_{9}, \boldsymbol{c}_{10}, \boldsymbol{c}_{11}, \boldsymbol{c}_{12}, \boldsymbol{c}_{13}, \boldsymbol{c}_{14}, \boldsymbol{c}_{15}\right]} \\
& \quad\left[\begin{array}{rrrrrrrrrrrrrrrrr}
0 & 1 & 0 & 0 & -1 & 0 & 0 & 1 & -1 & 1 & 1 & -1 & 1 & -1 & -1 \\
0 & 0 & 1 & 0 & 0 & -1 & 0 & 1 & 1 & -1 & 1 & -1 & -1 & 1 & -1 \\
0 & 0 & 0 & 1 & 0 & 0 & -1 & 1 & 1 & 1 & -1 & -1 & -1 & -1 & 1
\end{array}\right] .
\end{aligned}
$$

Here, we assume a binary miscible fluid mixture of $A$ - and $B$-species under the condition that the fraction of $B$-species is much smaller than that of $A$-species. Under this condition, the effect of $A-B$ collisions can be neglected compared to $A-A$ collisions. Also, the effect of $B-B$ collisions can be neglected in comparison

\section{R A F T February 15, 2002, 1:27pm D R A F T}


with $B-A$ collisions. Therefore, the evolution of the particle distribution function $f_{\sigma i}(\boldsymbol{x}, t)$ of $\sigma$-species $(\sigma=A, B)$ with velocity $\boldsymbol{c}_{i}$ at the point $\boldsymbol{x}$ and at time $t$ is computed by the following equation:

$$
\begin{aligned}
f_{\sigma i}\left(\boldsymbol{x}+\boldsymbol{c}_{i} \Delta x, t+\Delta t\right)-f_{\sigma i}(\boldsymbol{x}, t) & =-\frac{1}{\tau_{\sigma}}\left[f_{\sigma i}(\boldsymbol{x}, t)-f_{\sigma A i}^{\mathrm{eq}}(\boldsymbol{x}, t)\right] \\
& \text { for } i=1,2,3, \cdots, 15, \quad \sigma=A, B
\end{aligned}
$$

where $f_{\sigma A i}^{\mathrm{eq}}$ is an equilibrium distribution function for $\sigma$-species, $\tau_{\sigma}$ is a dimensionless single relaxation time and is of $O(1), \Delta x$ is a spacing of the cubic lattice, and $\Delta t$ is a time step during which the particles travel the lattice spacing. It is noted that $\Delta t=\operatorname{Sh} \Delta x$ where $\operatorname{Sh}(=U / c)$ is the Strouhal number.

The density $\rho_{A}$ of component $A$, the concentration $\rho_{B}$ of component $B$, and the flow velocity $\boldsymbol{u}_{A}$ of component $A$ are defined in terms of the particle distribution function as follows:

$$
\begin{aligned}
\rho_{\sigma} & =\sum_{i=1}^{15} f_{\sigma i} \text { for } \sigma=A, B \\
\boldsymbol{u}_{A} & =\frac{1}{\rho_{A}} \sum_{i=1}^{15} f_{A i} \boldsymbol{c}_{i} .
\end{aligned}
$$

As for the equilibrium distribution function for component $A$, it is noted that Eq. (2) for $\sigma=A$ has the same form as single-component isothermal LBM. Thus, the equilibrium distribution function $f_{A A i}^{\mathrm{eq}}$ is given by [4]

$$
\begin{array}{r}
f_{A A i}^{\mathrm{eq}}=E_{i} \rho_{A}\left[1+3 \boldsymbol{c}_{i} \cdot \boldsymbol{u}_{A}+\frac{9}{2}\left(\boldsymbol{c}_{i} \cdot \boldsymbol{u}_{A}\right)^{2}-\frac{3}{2} \boldsymbol{u}_{A} \cdot \boldsymbol{u}_{A}\right] \\
\text { for } i=1,2,3, \cdots, 15
\end{array}
$$

where $E_{1}=2 / 9, E_{i}=1 / 9$ for $i=2,3, \cdots, 7$, and $E_{i}=1 / 72$ for $i=8,9, \cdots, 15$. Moreover, in this model the pressure $p_{A}$ of component $A$ is related to the density $\rho_{A}$ by $[22]$

$$
p_{A}=\frac{1}{3} \rho_{A}
$$

\section{R A F T February 15, 2002, 1:27pm D R A F T}


As for the equilibrium distribution function for component $B$, on the other hand, we use the following equilibrium distribution function which contains the terms up to first order in flow velocity:

$$
f_{B A i}^{\mathrm{eq}}=E_{i} \rho_{B}\left(1+3 \boldsymbol{c}_{i} \cdot \boldsymbol{u}_{A}\right) \quad \text { for } \quad i=1,2,3, \cdots, 15 .
$$

The above distribution function is simpler than that of $[15,16]$. A similar equilibrium distribution function has been proposed in [17] with the hexagonal sevenvelocity model, but the accuracy of the method is not presented in detail. The appropriateness and the accuracy of the above equilibrium distribution function are verified theoretically and numerically in the following sections.

\subsection{Asymptotic Analysis}

Here, we are interested in the case of small Knudsen number with finite Reynolds number. Since Mach number $\mathrm{Ma}=U / c$, Reynolds number $\operatorname{Re}=U L / \nu(\nu$ is the kinematic viscosity of fluid), and Knudsen number Kn are related as Ma $\sim \mathrm{KnRe}$, it follows that $\mathrm{Ma}$ is of the same order of smallness as $\mathrm{Kn}$ in the case of finite Reynolds number. In addition, since $\mathrm{Sh}=U / c=\mathrm{Ma}$, the Strouhal number Sh is also of the order of Kn. It is also noted that $\Delta x$ is assumed to be of the same order as Kn. Considering this ordering, we carry out the asymptotic analysis for small Knudsen numbers according to [18-21]. As in [22], it is found that using Eqs. (2)-

(6) for $\sigma=A$ we can obtain the macroscopic flow velocities and pressure gradient of component $A$ for incompressible fluid with relative errors of $O\left(\varepsilon^{2}\right)$ where $\varepsilon$ is a modified Knudsen number which is of the same order as $\Delta x$.

Hereafter, we focus on the derivation of the governing equation for component B. First, performing a Taylor expansion of Eq. (2) for $\sigma=B$ up to $O\left[(\Delta x)^{4}\right]$ and dividing it by $\Delta x$, we have

$$
\left(\operatorname{Sh} \frac{\partial}{\partial t}+\boldsymbol{c}_{i} \cdot \nabla\right) f_{B i}+\frac{1}{2} \Delta x\left(\operatorname{Sh} \frac{\partial}{\partial t}+\boldsymbol{c}_{i} \cdot \nabla\right)^{2} f_{B i}+\frac{1}{6}(\Delta x)^{2}\left(\boldsymbol{c}_{i} \cdot \nabla\right)^{3} f_{B i}+O\left[(\Delta x)^{3}\right]
$$

\section{R A F T February 15, 2002, 1:27pm D R A F T}




$$
=-\frac{1}{\tau_{B} \Delta x}\left(f_{B i}-f_{B A i}^{\mathrm{eq}}\right)
$$

Next, we assume that the deviation of the distribution function from its equilibrium state at rest with the local concentration is of the same order as $\Delta x$ and put $f_{B i}$ in the form of series expansion of $\varepsilon$.

$$
f_{B i}=E_{i}\left(\rho_{B}^{(0)}+\varepsilon f_{B i}^{(1)}+\varepsilon^{2} f_{B i}^{(2)}+\varepsilon^{3} f_{B i}^{(3)}+\cdots\right) \quad \text { for } \quad i=1,2,3, \cdots, 15 .
$$

Corresponding to Eq. (9), the macroscopic variables are also expanded as follows:

$$
\begin{aligned}
& \rho_{B}=\rho_{B}^{(0)}+\varepsilon \rho_{B}^{(1)}+\varepsilon^{2} \rho_{B}^{(2)}+\varepsilon^{3} \rho_{B}^{(3)}+\cdots \\
& \boldsymbol{u}_{A}=\varepsilon \boldsymbol{u}_{A}^{(1)}+\varepsilon^{2} \boldsymbol{u}_{A}^{(2)}+\varepsilon^{3} \boldsymbol{u}_{A}^{(3)}+\cdots
\end{aligned}
$$

where

$$
\rho_{B}^{(m)}=\sum_{i=1}^{15} E_{i} f_{B i}^{(m)} \quad \text { for } m=1,2,3, \cdots
$$

It should be noted that the expansion of the flow velocity begins with the term of the order of $\varepsilon$, since Ma is of the order of Kn. Also, the equilibrium distribution function is expanded as follows:

$$
\begin{array}{r}
f_{B A i}^{\mathrm{eq}}=E_{i}\left(f_{B A i}^{\mathrm{eq}(0)}+\varepsilon f_{B A i}^{\mathrm{eq}(1)}+\varepsilon^{2} f_{B A i}^{\mathrm{eq}(2)}+\varepsilon^{3} f_{B A i}^{\mathrm{eq}(3)}+\cdots\right) \\
\text { for } i=1,2,3, \cdots, 15
\end{array}
$$

where

$$
\begin{aligned}
& f_{B A i}^{\mathrm{eq}(0)}=\rho_{B}^{(0)}, \\
& f_{B A i}^{\mathrm{eq}(1)}=\rho_{B}^{(1)}+3 \rho_{B}^{(0)} \boldsymbol{c}_{i} \cdot \boldsymbol{u}_{A}^{(1)}, \\
& f_{B A i}^{\mathrm{eq}(2)}=\rho_{B}^{(2)}+3 \rho_{B}^{(1)} \boldsymbol{c}_{i} \cdot \boldsymbol{u}_{A}^{(1)}+3 \rho_{B}^{(0)} \boldsymbol{c}_{i} \cdot \boldsymbol{u}_{A}^{(2)}, \\
& f_{B A i}^{\mathrm{eq}(3)}=\rho_{B}^{(3)}+3 \rho_{B}^{(2)} \boldsymbol{c}_{i} \cdot \boldsymbol{u}_{A}^{(1)}+3 \rho_{B}^{(1)} \boldsymbol{c}_{i} \cdot \boldsymbol{u}_{A}^{(2)}+3 \rho_{B}^{(0)} \boldsymbol{c}_{i} \cdot \boldsymbol{u}_{A}^{(3)} .
\end{aligned}
$$

\section{R A F T February 15, 2002, 1:27pm D R A F T}


We consider a moderately varying solution $\left[\partial f_{B i}^{(m)} / \partial t=O\left(f_{B i}^{(m)}\right)\right.$ and $\partial f_{B i}^{(m)} / \partial x_{\gamma}=$ $O\left(f_{B i}^{(m)}\right)$ with $m=1,2,3, \cdots$ and $\gamma=x, y, z$ (Subscript $\gamma$ represents Cartesian coordinates and the summation convention is used.)] of Eq. (2). Substituting Eqs. (9) and (12) into Eq. (8), we obtain the following simultaneous equations governing the component functions $f_{B i}^{(m)}(m=1,2,3, \cdots)$ of the velocity distribution functions $f_{B i}$ :

$$
\begin{aligned}
f_{B i}^{(1)}=f_{B A i}^{\mathrm{eq}(1)} & -\frac{\tau_{B} \Delta x}{\varepsilon} \boldsymbol{c}_{i} \cdot \nabla \rho_{B}^{(0)} \\
f_{B i}^{(2)}=f_{B A i}^{\mathrm{eq}(2)} & -\frac{\tau_{B} \Delta x}{\varepsilon} \boldsymbol{c}_{i} \cdot \nabla f_{B i}^{(1)} \\
& -\frac{\tau_{B} \Delta x}{\varepsilon}\left[\frac{\mathrm{Sh}}{\varepsilon} \frac{\partial}{\partial t}+\frac{1}{2} \frac{\Delta x}{\varepsilon}\left(\boldsymbol{c}_{i} \cdot \nabla\right)^{2}\right] \rho_{B}^{(0)}, \\
f_{B i}^{(3)}=f_{B A i}^{\mathrm{eq}(3)} & -\frac{\tau_{B} \Delta x}{\varepsilon} \boldsymbol{c}_{i} \cdot \nabla f_{B i}^{(2)} \\
& -\frac{\tau_{B} \Delta x}{\varepsilon}\left[\frac{\mathrm{Sh}}{\varepsilon} \frac{\partial}{\partial t}+\frac{1}{2} \frac{\Delta x}{\varepsilon}\left(\boldsymbol{c}_{i} \cdot \nabla\right)^{2}\right] f_{B i}^{(1)} \\
& -\tau_{B}\left(\frac{\Delta x}{\varepsilon}\right)^{2}\left(\boldsymbol{c}_{i} \cdot \nabla\right)\left[\frac{\mathrm{Sh}}{\varepsilon} \frac{\partial}{\partial t}+\frac{1}{6} \frac{\Delta x}{\varepsilon}\left(\boldsymbol{c}_{i} \cdot \nabla\right)^{2}\right] \rho_{B}^{(0)},
\end{aligned}
$$

which can also be written in the form of linear algebraic equations as follows:

$$
f_{B i}^{(m)}-\sum_{j=1}^{15} E_{j} f_{B j}^{(m)}=I h_{i}^{(m)} \quad \text { for } \quad m=1,2,3, \cdots,
$$

where $I h_{i}^{(m)}$ represents the inhomogeneous terms given by

$$
\begin{aligned}
I h_{i}^{(1)}= & 3 \rho_{B}^{(0)} \boldsymbol{c}_{i} \cdot \boldsymbol{u}_{A}^{(1)}-\frac{\tau_{B} \Delta x}{\varepsilon} \boldsymbol{c}_{i} \cdot \nabla \rho_{B}^{(0)} \\
I h_{i}^{(2)}= & 3 \rho_{B}^{(1)} \boldsymbol{c}_{i} \cdot \boldsymbol{u}_{A}^{(1)}+3 \rho_{B}^{(0)} \boldsymbol{c}_{i} \cdot \boldsymbol{u}_{A}^{(2)} \\
& \quad-\frac{\tau_{B} \Delta x}{\varepsilon} \boldsymbol{c}_{i} \cdot \nabla f_{B i}^{(1)}-\frac{\tau_{B} \Delta x}{\varepsilon}\left[\frac{\mathrm{Sh}}{\varepsilon} \frac{\partial}{\partial t}+\frac{1}{2} \frac{\Delta x}{\varepsilon}\left(\boldsymbol{c}_{i} \cdot \nabla\right)^{2}\right] \rho_{B}^{(0)}, \\
I h_{i}^{(3)}= & 3 \rho_{B}^{(2)} \boldsymbol{c}_{i} \cdot \boldsymbol{u}_{A}^{(1)}+3 \rho_{B}^{(1)} \boldsymbol{c}_{i} \cdot \boldsymbol{u}_{A}^{(2)}+3 \rho_{B}^{(0)} \boldsymbol{c}_{i} \cdot \boldsymbol{u}_{A}^{(3)}
\end{aligned}
$$

\section{R A F T February 15, 2002, 1:27pm D R A F T}




$$
\begin{aligned}
& -\frac{\tau_{B} \Delta x}{\varepsilon} \boldsymbol{c}_{i} \cdot \nabla f_{B i}^{(2)}-\frac{\tau_{B} \Delta x}{\varepsilon}\left[\frac{\mathrm{Sh}}{\varepsilon} \frac{\partial}{\partial t}+\frac{1}{2} \frac{\Delta x}{\varepsilon}\left(\boldsymbol{c}_{i} \cdot \nabla\right)^{2}\right] f_{B i}^{(1)} \\
& -\tau_{B}\left(\frac{\Delta x}{\varepsilon}\right)^{2}\left(\boldsymbol{c}_{i} \cdot \nabla\right)\left[\frac{\mathrm{Sh}}{\varepsilon} \frac{\partial}{\partial t}+\frac{1}{6} \frac{\Delta x}{\varepsilon}\left(\boldsymbol{c}_{i} \cdot \nabla\right)^{2}\right] \rho_{B}^{(0)}
\end{aligned}
$$

Equation (17) are inhomogeneous linear algebraic equations and have the same coefficient matrix in spite of $m$. As shown in Appendix B, the solvability conditions for Eq. (17) are given as follows:

$$
\sum_{i=1}^{15} E_{i} I h_{i}^{(m)}=0 \quad \text { for } \quad m=1,2,3, \cdots
$$

The solvability condition for $m=1$ is trivially satisfied. From the solvability condition (19) for $m=2$ we get

$$
\frac{\mathrm{Sh}}{\varepsilon} \frac{\partial \rho_{B}^{(0)}}{\partial t}+u_{A \gamma}^{(1)} \frac{\partial \rho_{B}^{(0)}}{\partial x_{\gamma}}=\frac{1}{3}\left(\tau_{B}-\frac{1}{2}\right) \frac{\Delta x}{\varepsilon} \frac{\partial^{2} \rho_{B}^{(0)}}{\partial x_{\gamma}^{2}} .
$$

From the solvability condition (19) for $m=3$ we get

$$
\frac{\mathrm{Sh}}{\varepsilon} \frac{\partial \rho_{B}^{(1)}}{\partial t}+u_{A \gamma}^{(1)} \frac{\partial \rho_{B}^{(1)}}{\partial x_{\gamma}}+u_{A \gamma}^{(2)} \frac{\partial \rho_{B}^{(0)}}{\partial x_{\gamma}}=\frac{1}{3}\left(\tau_{B}-\frac{1}{2}\right) \frac{\Delta x}{\varepsilon} \frac{\partial^{2} \rho_{B}^{(1)}}{\partial x_{\gamma}^{2}} .
$$

Multiplying Eq. (20) by $\varepsilon$ and Eq. (21) by $\varepsilon^{2}$ and taking the summation of the two equations, we obtain

$$
\begin{aligned}
\operatorname{Sh} \frac{\partial}{\partial t}\left(\rho_{B}^{(0)}+\varepsilon \rho_{B}^{(1)}\right)+ & \varepsilon u_{A \gamma}^{(1)} \frac{\partial}{\partial x_{\gamma}}\left(\rho_{B}^{(0)}+\varepsilon \rho_{B}^{(1)}\right)+\varepsilon^{2} u_{A \gamma}^{(2)} \frac{\partial \rho_{B}^{(0)}}{\partial x_{\gamma}} \\
& =\frac{1}{3}\left(\tau_{B}-\frac{1}{2}\right) \Delta x \frac{\partial^{2}}{\partial x_{\gamma}^{2}}\left(\rho_{B}^{(0)}+\varepsilon \rho_{B}^{(1)}\right) .
\end{aligned}
$$

Equation (22) corresponds to the convection-diffusion equation for component $B$ in a binary miscible fluid mixture. Therefore, it is found that using Eqs. (2)-(4) and (7) for $\sigma=B$ we can obtain the concentration of component $B$ in a binary miscible fluid mixture with relative errors of $O\left(\varepsilon^{2}\right)$.

\section{R A F T February 15, 2002, 1:27pm D R A F T}


The dimensionless kinematic viscosity $\nu$ of the fluid and the dimensionless mass diffusivity $D_{B A}$ in the binary miscible fluid mixture are given by [22]

$$
\begin{aligned}
\nu & =\frac{1}{3}\left(\tau_{A}-\frac{1}{2}\right) \Delta x, \\
D_{B A} & =\frac{1}{3}\left(\tau_{B}-\frac{1}{2}\right) \Delta x .
\end{aligned}
$$

Then the Schmidt number Sc becomes

$$
\mathrm{Sc}=\frac{\nu}{D_{B A}}=\frac{2 \tau_{A}-1}{2 \tau_{B}-1} .
$$

\subsection{Numerical Results}

To demonstrate the validity of the proposed method, we calculate a steady diffusion problem between two parallel walls. Here we use the two-dimensional ninevelocity model $[4,23]$ for simplicity. The nine-velocity model has the following velocity vectors: $\boldsymbol{c}_{1}=\mathbf{0}, \boldsymbol{c}_{i}=[\cos (\pi(i-2) / 2), \sin (\pi(i-2) / 2)]$ for $i=2,3,4,5$,

and $\boldsymbol{c}_{i}=\left[\cos \left(\pi\left(i-\frac{11}{2}\right) / 2\right), \sin \left(\pi\left(i-\frac{11}{2}\right) / 2\right)\right]$ for $i=6,7,8,9$. The basic theory and equations for the nine-velocity model are the same as those for the fifteen-velocity model except that the coefficients $E_{i}$ are $E_{1}=4 / 9, E_{i}=1 / 9$ for $i=2,3,4,5$, and $E_{i}=1 / 36$ for $i=6,7,8,9$.

A square domain with the sides of length $L$ is divided into square lattices with the spacing of $\Delta x$. The lower and upper walls are located at $y=0$ and $y=1$, respectively. The two walls are assumed to be porous ones and a constant normal flow $v_{A 0}$ of component $A$ is injected through the lower wall and is removed from the upper wall. The concentration of component $B$ at the lower and upper walls is maintained with $\rho_{B \mathrm{~L}}$ and $\rho_{B \mathrm{U}}$, respectively. In this problem, $\rho_{B \mathrm{U}}$ is assumed higher than $\rho_{B \mathrm{~L}}$; it follows that $B$-species diffuses counter to the flow of $A$-species. The governing equation for this problem becomes

$$
v_{A 0} \frac{d \Pi_{B}}{d y}=D_{B A} \frac{d^{2} \Pi_{B}}{d y^{2}}
$$

\section{R A F T February 15, 2002, 1:27pm D R A F T}


where $\Pi_{B}$ is a normalized concentration defined as follows:

$$
\Pi_{B}=\frac{\rho_{B}-\rho_{B \mathrm{~L}}}{\rho_{B \mathrm{U}}-\rho_{B \mathrm{~L}}} .
$$

The analytical solution $\Pi_{B}^{*}$ is given by

$$
\Pi_{B}^{*}=\frac{\exp \left(v_{A 0} y / D_{B A}\right)-1}{\exp \left(v_{A 0} / D_{B A}\right)-1}
$$

In the following calculations, we keep the condition of $v_{A 0} / D_{B A}=4$. The periodic boundary condition is imposed in the $x$-direction. On the lower and upper walls the boundary condition with constant concentration of component $B$ is used (see Appendix C). We carried out calculations with $\Delta x=1 / 20,1 / 40$, and $1 / 80$ and with $\tau_{B}=1.1,1.4$, and 1.7. Figure 1 shows the calculated concentration profile with $\Delta x=0.05$ and $\tau_{B}=1.1$. The solid line and the closed circles indicate the analytical solution and the calculated results, respectively. It is seen that the results agree well with the analytical solution. Next, the errors of the calculated results from the analytical solution with various $\Delta x$ and $\tau_{B}$ are examined. As stated in [22], the errors in this problem are proportional to $(\Delta x)^{2}$ alone as long as $v_{A 0} / D_{B A}$ is kept at a constant value. Table 1 presents the error norms $E r_{1}=\sum_{y}\left|\Pi_{B}-\Pi_{B}^{*}\right| / \sum_{y}\left|\Pi_{B}^{*}\right|$ and $E r_{2}=\sqrt{\sum_{y}\left(\Pi_{B}-\Pi_{B}^{*}\right)^{2}} / \sqrt{\sum_{y}\left(\Pi_{B}^{*}\right)^{2}}$ where the sums are taken over the same 21 nodes between the walls for all cases. It is clearly found that the errors decrease in proportion to $(\Delta x)^{2}$ regardless of $\tau_{B}$ and the present LBM is a second-order scheme.

\section{APPLICATION TO THERMAL FLUID SYSTEMS}

\subsection{Basic Equation}

The present LBM for the binary miscible fluid mixture can also be applied to thermal fluid systems by taking advantage of the formal analogy between heat and mass transfer. In the following, the concentration field of component $B$ is regarded

\section{R A F T February 15, 2002, 1:27pm D R A F T}


as the temperature field of component $A$ and the subscript $B$ of the variables for component $B$ is replaced by $T$ denoting temperature. As for the motion of component $A$, the Boussinesq approximation is used for the gravitational term and a buoyancy force proportional to the temperature difference is included. Here the buoyancy force is assumed to be acting in the $y$-direction alone. Then the evolution of particle distribution functions for fluid $A$ and temperature $T$ is written as follows $[24]:$

$$
\begin{array}{r}
f_{\sigma i}\left(\boldsymbol{x}+\boldsymbol{c}_{i} \Delta x, t+\Delta t\right)-f_{\sigma i}(\boldsymbol{x}, t)=-\frac{1}{\tau_{\sigma}}\left[f_{\sigma i}(\boldsymbol{x}, t)-f_{\sigma A i}^{\mathrm{eq}}(\boldsymbol{x}, t)\right] \\
+3 E_{i} g \beta\left(T-T^{*}\right) c_{i y} \Delta x \delta_{\sigma A}
\end{array}
$$

where $g$ is the gravitational acceleration, $\beta$ is the volumetric expansion coefficient, $T^{*}$ is a reference temperature, and $\delta_{\sigma A}$ is the Kronecker delta. Note that $g \beta$ is of $O\left(\varepsilon^{2}\right)$. The equilibrium distribution functions $f_{A A}^{\mathrm{eq}}$ and $f_{T A}^{\mathrm{eq}}$ are given by Eqs. (5) and $(7)$, respectively. Also, the density $\rho$, the temperature $T$ (corresponding to $\left.\rho_{B}\right)$, the flow velocity $\boldsymbol{u}$, and the pressure $p$ of the fluid are defined as Eqs. (3), (4), and (6).

\subsection{Governing Equation}

Here, the governing equations for the thermal fluid systems are derived. As in the case of the LBM for the binary miscible fluid mixture, the asymptotic analysis is applied to Eq. (29). It is shown from the results that $\boldsymbol{u}=\varepsilon \boldsymbol{u}^{(1)}+\varepsilon^{2} \boldsymbol{u}^{(2)}+O\left(\varepsilon^{3}\right)$, $p=\varepsilon^{2} p^{(2)}+\varepsilon^{3} p^{(3)}+O\left(\varepsilon^{4}\right)$, and $T=T^{(0)}+\varepsilon T^{(1)}+O\left(\varepsilon^{2}\right)$ satisfy

$$
\begin{gathered}
\frac{\partial u_{\gamma}}{\partial x_{\gamma}}=0 \\
\operatorname{Sh} \frac{\partial u_{\gamma}}{\partial t}+u_{\delta} \frac{\partial u_{\gamma}}{\partial x_{\delta}}=-\frac{\partial p}{\partial x_{\gamma}}+\frac{1}{3}\left(\tau_{A}-\frac{1}{2}\right) \Delta x \frac{\partial^{2} u_{\gamma}}{\partial x_{\delta}^{2}}+g \beta\left(T-T^{*}\right) \delta_{\gamma y},
\end{gathered}
$$

\section{R A F T February 15, 2002, 1:27pm D R A F T}




$$
\operatorname{Sh} \frac{\partial T}{\partial t}+u_{\gamma} \frac{\partial T}{\partial x_{\gamma}}=\frac{1}{3}\left(\tau_{T}-\frac{1}{2}\right) \Delta x \frac{\partial^{2} T}{\partial x_{\gamma}^{2}}
$$

where $\delta_{\gamma y}$ is the Kronecker delta. It is noted that the summation convention is used for the subscript $\gamma$ and $\delta(\gamma, \delta=x, y, z)$ in Eqs. (30)-(32). Equations (30), (31), and (32) correspond to the continuity equation, the Navier-Stokes equations, and the convection-diffusion equation for the temperature for incompressible fluid, respectively.

The dimensionless kinematic viscosity $\nu$ and the dimensionless thermal diffusivity $\alpha$ (corresponding to $D_{B A}$ ) of the fluid are given by Eqs. (23) and (24), respectively. Then the Prandtl number Pr and the Rayleigh number Ra are determined as follows:

$$
\begin{gathered}
\operatorname{Pr}=\frac{\nu}{\alpha}=\frac{2 \tau_{A}-1}{2 \tau_{T}-1}, \\
\operatorname{Ra}=\frac{36 g \beta \Delta T}{\left(2 \tau_{A}-1\right)\left(2 \tau_{T}-1\right)(\Delta x)^{2}},
\end{gathered}
$$

where $\Delta T$ is a characteristic temperature difference.

\subsection{Numerical Results}

As a typical example of thermal fluid systems, Rayleigh-Bénard convection is calculated by the present LBM. Here is also used the nine-velocity model for simplicity. A rectangular domain with height $L$ and width $2 L$ is divided into square lattices with the spacing of $\Delta x$. The temperature $T_{\mathrm{L}}$ on the lower wall at $y=0$ is kept higher than the temperature $T_{\mathrm{U}}$ on the upper wall at $y=1 . T^{*}$ is given by $[15,16]$

$$
T^{*}=T_{\mathrm{L}}-y \Delta T
$$

where $\Delta T=T_{\mathrm{L}}-T_{\mathrm{U}}$. Note that Eq. (35) leads to cancel the effect of hydrostatic pressure due to the buoyancy force. Hence, in the conductive state the buoyancy force vanishes and the pressure field is homogeneous $[15,16]$. The periodic boundary condition is used in the $x$-direction. The no-slip boundary condition with constant

\section{R A F T February 15, 2002, 1:27pm D R A F T}


temperature is used on the lower and upper walls. (See Appendix $\mathrm{C}$ with $v_{A 0}=0$.) Computational conditions are $\Delta x=1 / 50$ and $\operatorname{Pr}=0.71$, and the other parameters are changed so that the Rayleigh number Ra ranges from 1,650 to 100,000.

We first evaluate the critical Rayleigh number $\mathrm{Ra}_{\mathrm{c}}$. The calculations are started from the static conductive state at several different Rayleigh numbers close to $\mathrm{Ra}_{\mathrm{c}}$. An initial small perturbation in a sine wave is applied to the temperature field. The amplitude of the sine wave is set to be $5 \times 10^{-3}$ for all cases. Figure 2 shows the time histories of the maximum velocities in the $y$-direction at $\mathrm{Ra}=$ $1,650,1,680,1,700,1,720,1,750$, and 1,800 . The initial disturbance is found to either grow or decay exponentially according to the Rayleigh numbers. Then the growth rate, which is defined as the rate of increase in the maximum velocity in the $y$-direction during one time step, is calculated and interpolated to obtain the Rayleigh number corresponding to zero growth rate. Figure 3 shows the calculated growth rates plotted against the Rayleigh numbers. It should be noted that negative value of the growth rate indicates the rate of decrease in the maximum vertical velocity. In Fig. 3, the solid straight line is drawn through the data points using the least-squares fitting and the intersection with the horizontal axis gives the critical Rayleigh number. The calculated critical Rayleigh number is 1708.48 and agrees well with the theoretical value of 1707.76 obtained by linear stability theory [25] within $0.042 \%$.

We next present the calculated results at higher Rayleigh numbers. Figure 4 shows typical velocity vectors and temperature fields in final steady-states at $\mathrm{Ra}=5,000,20,000$, and 50,000. In Fig. 4, the length of each velocity vector is normalized by the maximum velocity in the $y$-direction at $\mathrm{Ra}=50,000$. It is seen that as the Rayleigh number increases, the mixing of the hot and cold fluids is enhanced and the temperature gradients near the lower and upper walls become steeper. It is found that our results are qualitatively reasonable compared to the results by Shan [15] and by He et al. [16]. The Nusselt number can be calculated

\section{R A F T February 15, 2002, 1:27pm D R A F T}


by the following equation [16]:

$$
\mathrm{Nu}=1+\frac{\left\langle u_{y} T\right\rangle}{\alpha \Delta T}
$$

where $u_{y}$ is the flow velocity in the $y$-direction and $\langle\cdot\rangle$ denotes the average over the whole flow domain. Figure 5 shows the calculated relationship between the Nusselt number and the Rayleigh number. In Fig. 5, the results by Clever and Busse [26] and the empirical formula $\mathrm{Nu}=1.56\left(\mathrm{Ra} / \mathrm{Ra}_{\mathrm{c}}\right)^{0.296}[16]$ are also shown for comparison. It is seen that our results agree fairly well with those by Clever and Busse at various Rayleigh numbers up to 100,000. Shan [15] and He et al. [16] calculate the same problem, but their calculations slightly underestimate the heat transfer at Rayleigh numbers higher than 20,000. Therefore, it is found that the present LBM is more accurate than their thermal LBMs at high Rayleigh numbers.

\section{CONCLUDING REMARKS}

We have proposed an LBM for an isothermal binary miscible fluid mixture. Applying the asymptotic theory proposed by Sone [18-21] to the present LBM, we found that the concentration of the diffusing component in the mixture can be obtained with relative errors of $O\left(\varepsilon^{2}\right)$ where $\varepsilon$ is a modified Knudsen number which is of the same order as the lattice spacing. The proposed LBM can be applied to thermal fluid systems. In two problems, diffusion between two parallel walls and Rayleigh-Bénard convection, we found that the present LBM is useful for the simulation of fluid flows with heat and mass transfer.

\section{APPENDIX A}

\section{Definition of Non-Dimensional Variables}

As in [22], we use the following non-dimensional variables defined by a characteristic length $L$, a characteristic particle speed $c$, a characteristic time scale $t_{0}=L / U$

\section{R A F T February 15, 2002, 1:27pm D R A F T}


where $U$ is a characteristic flow speed, a reference density $\rho_{A 0}$, a reference concentration $\rho_{B 0}$, and a reference temperature $T_{0}$ :

$$
\left.\begin{array}{lll}
\hat{\boldsymbol{c}}_{i}=\boldsymbol{c}_{i} / c, & \hat{\boldsymbol{x}}=\boldsymbol{x} / L, & \hat{t}=t / t_{0}, \\
\hat{f}_{A i}=f_{A i} / \rho_{A 0}, & \hat{f}_{B i}=f_{B i} / \rho_{B 0}, & \\
\hat{\rho}_{A}=\rho_{A} / \rho_{A 0}, & \hat{\rho}_{B}=\rho_{B} / \rho_{B 0}, & \\
\hat{\boldsymbol{u}}_{A}=\boldsymbol{u}_{A} / c, & \hat{p}_{A}=p_{A} /\left(\rho_{A 0} c^{2}\right), & \\
\hat{g}=g L / c^{2}, & \hat{\beta}=\beta T_{0}, & \hat{T}=T / T_{0}, \\
\hat{\nu}=\nu /(c L), & \hat{D}_{B A}=D_{B A} /(c L), & \hat{\alpha}=\alpha /(c L),
\end{array}\right\}
$$

where the subscript $A$ and $B$ represent the variables for $A$ - and $B$-species, respectively. The dimensional variables in Eq. (A.1) are as follows: $\boldsymbol{c}_{i}$ is the particle velocity, $\boldsymbol{x}$ is the coordinates, $t$ is the time, $f_{A i}$ and $f_{B i}$ are the particle distribution functions, $\rho_{A}$ is the density, $\rho_{B}$ is the concentration, $\boldsymbol{u}_{A}$ is the flow velocity, $p_{A}$ is the pressure, $g$ is the gravitational acceleration, $\beta$ is the volumetric expansion coefficient, $T$ is the temperature, $\nu$ is the kinematic viscosity, $D_{B A}$ is the mass diffusivity, and $\alpha$ is the thermal diffusivity.

\section{APPENDIX B}

Solvability Conditions of Eq. (17)

The linear algebraic Eq. (17) can be written as

$$
A \boldsymbol{y}=\boldsymbol{b}
$$

\section{R A F T February 15, 2002, 1:27pm D R A F T}


with

$$
A=\frac{1}{72}\left[\begin{array}{rrrrrrrrrrrrrrrr}
56 & -8 & -8 & -8 & -8 & -8 & -8 & -1 & -1 & -1 & -1 & -1 & -1 & -1 & -1 \\
-16 & 64 & -8 & -8 & -8 & -8 & -8 & -1 & 1 & -1 & -1 & -1 & -1 & -1 & -1 \\
-16 & -8 & 64 & -8 & -8 & -8 & -8 & -1 & -1 & -1 & -1 & -1 & -1 & -1 & -1 \\
-16 & -8 & -8 & 64 & -8 & -8 & -8 & -1 & -1 & -1 & -1 & -1 & -1 & -1 & -1 \\
-16 & -8 & -8 & -8 & 64 & -8 & -8 & -1 & -1 & -1 & -1 & -1 & -1 & -1 & -1 \\
-16 & -8 & -8 & -8 & -8 & 64 & -8 & -1 & -1 & -1 & -1 & -1 & -1 & -1 & -1 \\
-16 & -8 & -8 & -8 & -8 & -8 & 64 & -1 & -1 & -1 & -1 & -1 & -1 & -1 & -1 \\
-16 & -8 & -8 & -8 & -8 & -8 & -8 & -8 & 71 & -1 & -1 & -1 & -1 & -1 & -1 & -1 \\
-16 & -8 & -8 & -8 & -8 & -8 & -8 & -1 & -1 & -1 & 71 & -1 & -1 & -1 & -1 \\
-16 & -8 & -8 & -8 & -8 & -8 & -8 & -1 & -1 & -1 & -1 & 71 & -1 & -1 & -1 \\
-16 & -8 & -8 & -8 & -8 & -8 & -8 & -1 & -1 & -1 & -1 & -1 & 71 & -1 & -1 \\
-16 & -8 & -8 & -8 & -8 & -8 & -8 & -1 & -1 & -1 & -1 & -1 & -1 & 71 & -1 \\
-16 & -8 & -8 & -8 & -8 & -8 & -1 & -1 & -1 & -1 & -1 & -1 & -1 & 71
\end{array}\right],
$$

where

$$
\begin{array}{r}
\boldsymbol{y}=\left[f_{1}^{(m)}, f_{2}^{(m)}, f_{3}^{(m)}, f_{4}^{(m)}, f_{5}^{(m)}, f_{6}^{(m)}, f_{7}^{(m)}, f_{8}^{(m)},\right. \\
\left.f_{9}^{(m)}, f_{10}^{(m)}, f_{11}^{(m)}, f_{12}^{(m)}, f_{13}^{(m)}, f_{14}^{(m)}, f_{15}^{(m)}\right]^{\mathrm{T}} \\
\text { with } m \geq 1,
\end{array}
$$

and

$$
\begin{gathered}
\boldsymbol{b}=\left[I h_{1}^{(m)}, I h_{2}^{(m)}, I h_{3}^{(m)}, I h_{4}^{(m)}, I h_{5}^{(m)}, I h_{6}^{(m)}, I h_{7}^{(m)}, I h_{8}^{(m)},\right. \\
\left.I h_{9}^{(m)}, I h_{10}^{(m)}, I h_{11}^{(m)}, I h_{12}^{(m)}, I h_{13}^{(m)}, I h_{14}^{(m)}, I h_{15}^{(m)}\right]^{\mathrm{T}} \\
\text { with } \quad m \geq 1 .
\end{gathered}
$$

D R A F T February 15, 2002, 1:27pm D R A F T 
It is easily verified that $\operatorname{rank}\left(A^{\mathrm{T}}\right)=14$ and the equation $A^{\mathrm{T}} \boldsymbol{y}^{\prime}=\mathbf{0}$ has one nontrivial solution $\boldsymbol{y}^{\prime}=[1,1,1,1,1,1,1,1,1,1,1,1,1,1,1]^{\mathrm{T}}$. Also, multiplying each row vector of the matrix $A$ by $E_{i}$, we get a symmetric matrix. Thus, from the fundamental theorem of linear algebra [27] we obtain the solvability conditions Eq. (19) for Eq. (17).

\section{APPENDIX C}

\section{Boundary Condition}

We present boundary condition for component $B$ with constant concentration at a wall. On the lower wall at $y=0$, for example, $f_{B 3}, f_{B 6}$, and $f_{B 7}$ are unknown. As in [28], the unknown distribution functions are assumed to be the equilibrium distribution functions given by Eq. (7) with a parameter $\rho_{B}^{\prime}$ as follows:

$$
f_{B i}=E_{i} \rho_{B}^{\prime}\left(1+3 v_{A 0}\right) \quad \text { for } \quad i=3,6,7
$$

where $v_{A 0}$ is the flow velocity of component $A$ in the $y$-direction. The unknown parameter $\rho_{B}^{\prime}$ is determined so that the concentration of component $B$ at the wall is equal to a given value of $\rho_{B \mathrm{~L}}$. Substituting Eq. (C.1) and the known distribution functions into Eq. (3) for $\sigma=B$, the unknown parameter $\rho_{B}^{\prime}$ is specified as follows:

$$
\rho_{B}^{\prime}=\frac{12}{2+3 v_{A 0}}\left(\rho_{B \mathrm{~L}}-f_{B 1}-f_{B 2}-f_{B 4}-f_{B 5}-f_{B 8}-f_{B 9}\right)
$$

The same method is used on the upper wall at $y=1$.

\section{ACKNOWLEDGMENTS}

This research was partly supported by the Grant-in-Aid (No. 10650172) for Scientific Research from the Ministry of Education, Culture, Sports, Science and Technology in Japan. The authors thank the anonymous referees for helpful suggestions on the manuscript.

\section{R A F T February 15, 2002, 1:27pm D R A F T}




\section{REFERENCES}

1. G. McNamara and G. Zanetti, Use of the Boltzmann equation to simulate lattice-gas automata, Phys. Rev. Lett. 61, 2332 (1988).

2. F. Higuera and J. Jimenez, Boltzmann approach to lattice gas simulations, Europhys. Lett. 9, $663(1989)$.

3. S. Chen, H. Chen, D. Martinez, and W. H. Matthaeus, Lattice Boltzmann model for simulation of magnetohydrodynamics, Phys. Rev. Lett. 67, 3776 (1991).

4. Y. H. Qian, D. d'Humières, and P. Lallemand, Lattice BGK models for Navier-Stokes equation, Europhys. Lett. 17, 479 (1992).

5. A. K. Gunstensen, D. H. Rothman, S. Zaleski, and G. Zanetti, Lattice Boltzmann model of immiscible fluids, Phys. Rev. A 43, 4320 (1991).

6. D. H. Rothman and J. M. Keller, Immiscible cellular-automaton fluids, J. Stat. Phys. 52, 1119 (1988).

7. R. Holme and D. H. Rothman, Lattice-gas and lattice-Boltzmann models of miscible fluids, J. Stat. Phys. 68, 409 (1992).

8. E. G. Flekkøy, Lattice Bhatnagar-Gross-Krook models for miscible fluids, Phys. Rev. E 47, 4247 (1993).

9. X. Shan and G. Doolen, Multicomponent lattice-Boltzmann model with interparticle interaction, J. Stat. Phys. 81, 379 (1995).

10. X. Shan and G. Doolen, Diffusion in a multicomponent lattice Boltzmann equation model, Phys. Rev. E 54, 3614 (1996).

11. G. R. McNamara, A. L. Garcia, and B. J. Alder, A hydrodynamically correct thermal lattice Boltzmann model, J. Stat. Phys. 87, 1111 (1997).

12. F. J. Alexander, S. Chen, and J. D. Sterling, Lattice Boltzmann thermohydrodynamics, Phys. Rev. E 47, R2249 (1993).

13. Y. H. Qian, Simulating thermohydrodynamics with lattice BGK models, J. Sci. Comput. 8, 231 (1993).

\section{R A F T February 15, 2002, 1:27pm D R A F T}


14. Y. Chen, H. Ohashi, and M. Akiyama, Two-parameter thermal lattice BGK model with a controllable Prandtl number, J. Sci. Comput. 12, 169 (1997).

15. X. Shan, Simulation of Rayleigh-Bénard convection using a lattice Boltzmann method, Phys. Rev. E 55, 2780 (1997).

16. X. He, S. Chen, and G. D. Doolen, A novel thermal model for the lattice Boltzmann method in incompressible limit, J. Comput. Phys. 146, 282 (1998).

17. D. H. Rothman and S. Zaleski, Lattice-Gas Cellular Automata, (Cambridge University Press, Cambridge, 1997), p. 97.

18. Y. Sone, Asymptotic theory of flow of rarefied gas over a smooth boundary II, in Rarefied Gas Dynamics, edited by D. Dini (Editrice Tecnico Scientifica, Pisa, 1971), Vol. 2, p. 737.

19. Y. Sone, Asymptotic theory of a steady flow of a rarefied gas past bodies for small Knudsen numbers, in Advances in Kinetic Theory and Continuum Mechanics, edited by R. Gatignol and Soubbaramayer (Springer-Verlag, Berlin, 1991), p. 19.

20. Y. Sone, Analytical and numerical studies of rarefied gas flows on the basis of the Boltzmann equation for hard-sphere molecules, in Rarefied Gas Dynamics, edited by A. E. Beylich (VCH, Weinheim, 1991), p. 489.

21. Y. Sone and K. Aoki, Molecular Gas Dynamics (Asakura, Tokyo, 1994), Chap. 3 (in Japanese).

22. T. Inamuro, M. Yoshino, and F. Ogino, Accuracy of the lattice Boltzmann method for small Knudsen number with finite Reynolds number, Phys. Fluids 9, 3535 (1997).

23. B. T. Nadiga, A study of multi-speed discrete-velocity gases, Ph. D. thesis, California Institute of Technology, 1992.

24. J. M. Buick and C. A. Greated, Lattice Boltzmann modeling of interfacial gravity waves, Phys. Fluids 10, 1490 (1998).

25. W. H. Reid and D. L. Harris, Some further results on the Bénard Problem, Phys. Fluids 1, 102 (1958).

26. R. M. Clever and F. H. Busse, Transition to time-dependent convection, J. Fluid Mech. 65, 625 (1974).

\section{R A F T February 15, 2002, 1:27pm D R A F T}


27. G. Strang, Linear Algebra and Its Applications, (Academic Press, Inc., New York, 1976), p. 81.

28. T. Inamuro, M. Yoshino, and F. Ogino, A non-slip boundary condition for lattice Boltzmann simulations, Phys. Fluids 7, 2928 (1995); Erratum: 8, 1124 (1996).

D R A F T February 15, 2002, 1:27pm D R A F T 
TABLE 1. Error norms of diffusion problem between two parallel walls.

\begin{tabular}{lccc}
\hline$\Delta x$ & $\tau_{B}$ & $E r_{1}$ & $E r_{2}$ \\
\hline 0.05 & $1.1,1.4$, and 1.7 & $2.254 \times 10^{-3}$ & $1.790 \times 10^{-3}$ \\
0.025 & $1.1,1.4$, and 1.7 & $5.617 \times 10^{-4}$ & $4.459 \times 10^{-4}$ \\
0.0125 & $1.1,1.4$, and 1.7 & $1.403 \times 10^{-4}$ & $1.114 \times 10^{-4}$ \\
\hline
\end{tabular}

D R A F T February 15, 2002, 1:27pm D R A F T 


\section{FIGURES}

FIG. 1. Calculated concentration profile $\Pi_{B}$ of a binary fluid diffusion problem between parallel walls with $\Delta x=0.05$ and $\tau_{B}=1.1$.

FIG. 2. Time histories of the maximum vertical velocity at different Rayleigh numbers close to critical value of $\mathrm{Ra}_{\mathrm{c}}$.

FIG. 3. Growth rates of the instability against the Rayleigh numbers. The closed circles indicate the results obtained from the time histories of the maximum vertical velocity, and the solid straight line is drawn through the data points using the least-squares fitting.

FIG. 4. Calculated velocity vectors (left) and normalized temperature $\left(T-T_{\mathrm{U}}\right) /\left(T_{\mathrm{L}}-\right.$ $\left.T_{\mathrm{U}}\right)$ where $T_{\mathrm{L}}$ and $T_{\mathrm{U}}$ are the temperatures on the lower and upper walls, respectively (right), in final steady-states at different Rayleigh numbers. (a) Ra=5,000, (b) Ra=20,000, and (c) $\mathrm{Ra}=50,000 . u_{y, \max }$ is the maximum velocity in the $y$-direction at $\mathrm{Ra}=50,000$ and temperature contour interval is 0.05 .

FIG. 5. Calculated relationship between the Nusselt number and the Rayleigh number. The results by Clever and Busse $[26]$ and the empirical formula $\mathrm{Nu}=1.56(\mathrm{Ra} / \mathrm{Ra})^{0.296}$ [16] where $\mathrm{Ra}_{\mathrm{c}}$ is the critical Rayleigh number are also shown for comparison.

\section{R A F T February 15, 2002, 1:27pm D R A F T}




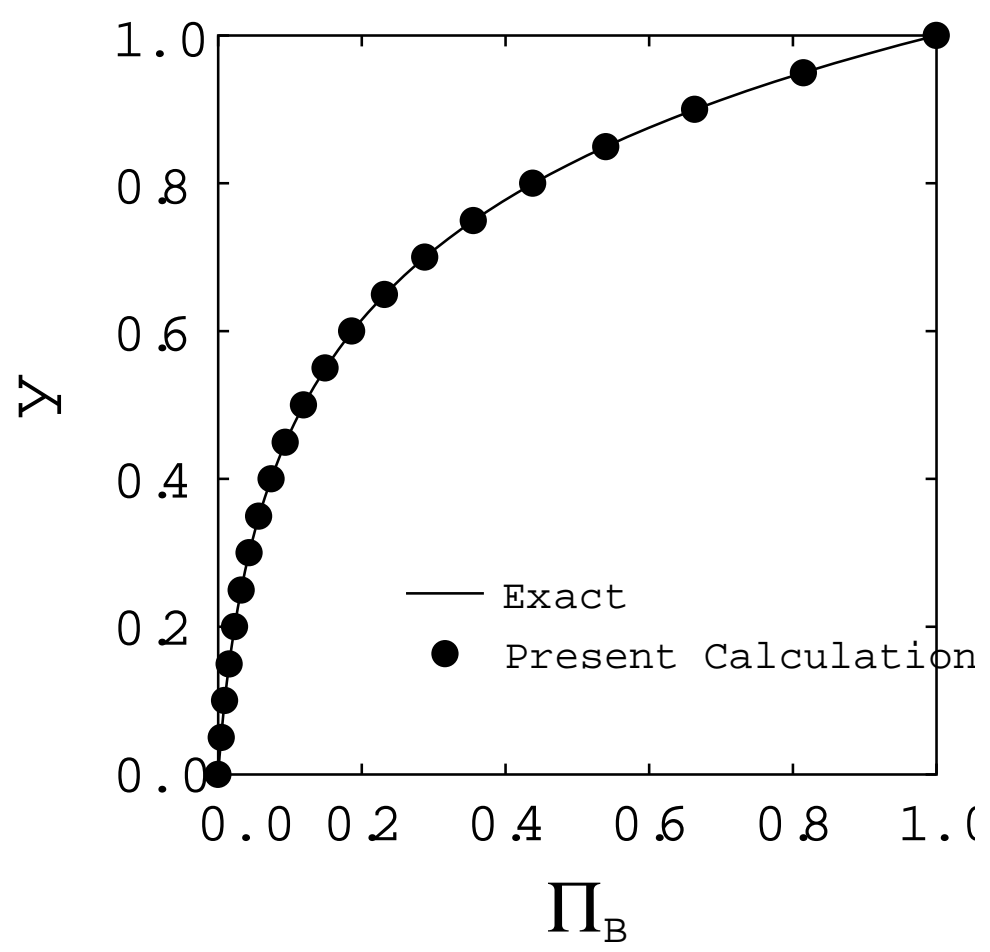

FIG. 1. Calculated concentration profile $\Pi_{B}$ of a binary fluid diffusion problem between parallel walls with $\Delta x=0.05$ and $\tau_{B}=1.1$.

D R A F T February 15, 2002, 1:27pm D R A F T 


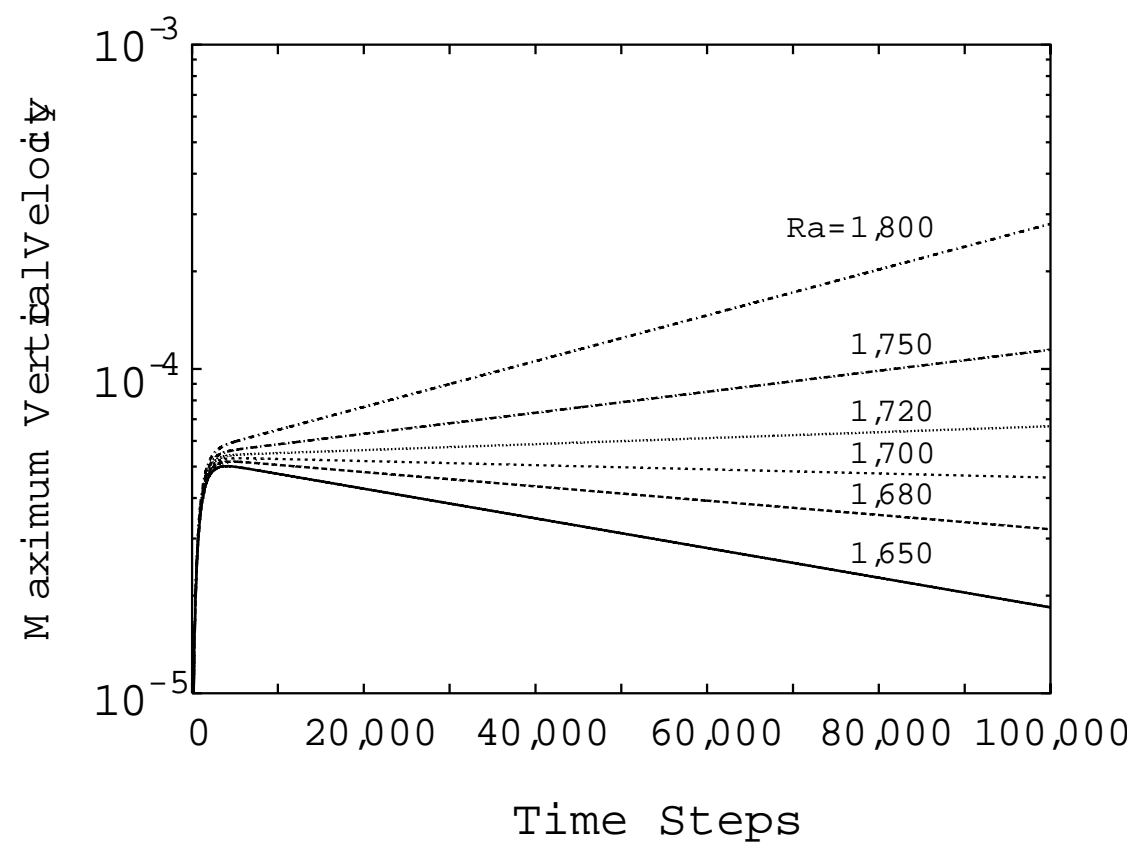

FIG. 2. Time histories of the maximum vertical velocity at different Rayleigh numbers close to critical value of $\mathrm{Ra}_{\mathrm{c}}$.

D R A F T February 15, 2002, 1:27pm D R A F T 


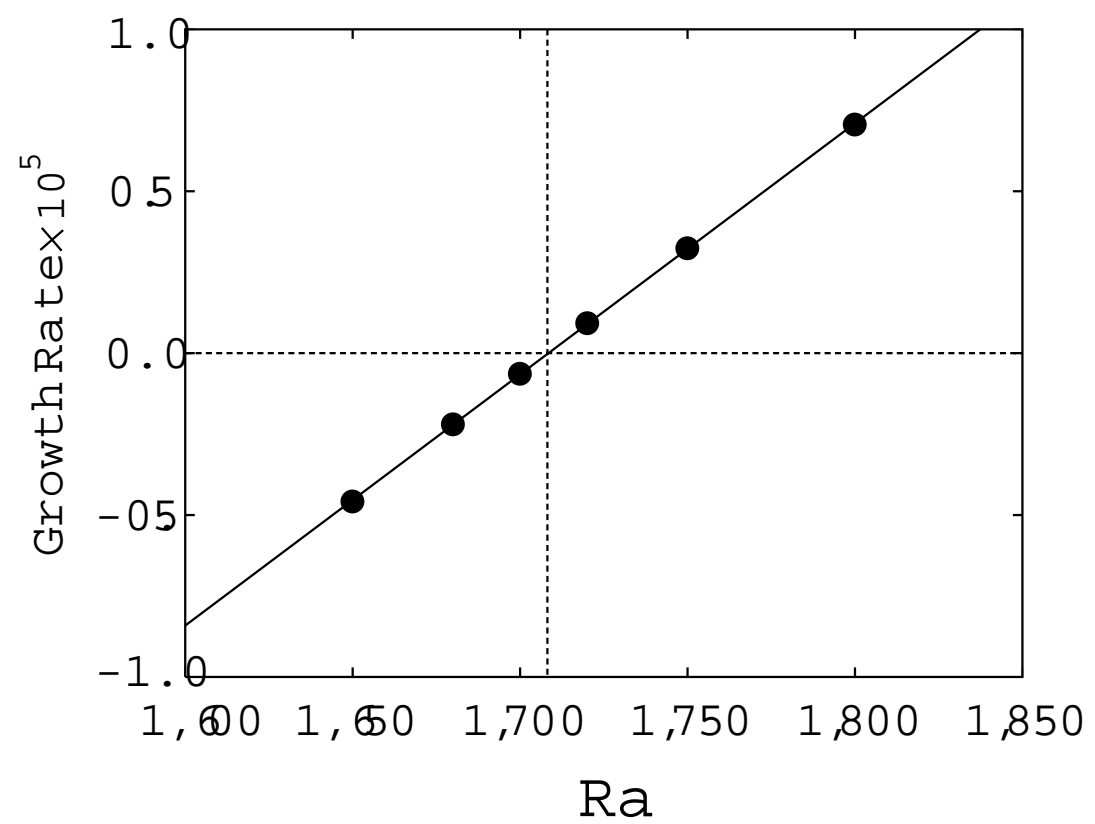

FIG. 3. Growth rates of the instability against the Rayleigh numbers. The closed circles indicate the results obtained from the time histories of the maximum vertical velocity, and the solid straight line is drawn through the data points using the least-squares fitting.

D R A F T February 15, 2002, 1:27pm D R A F T 

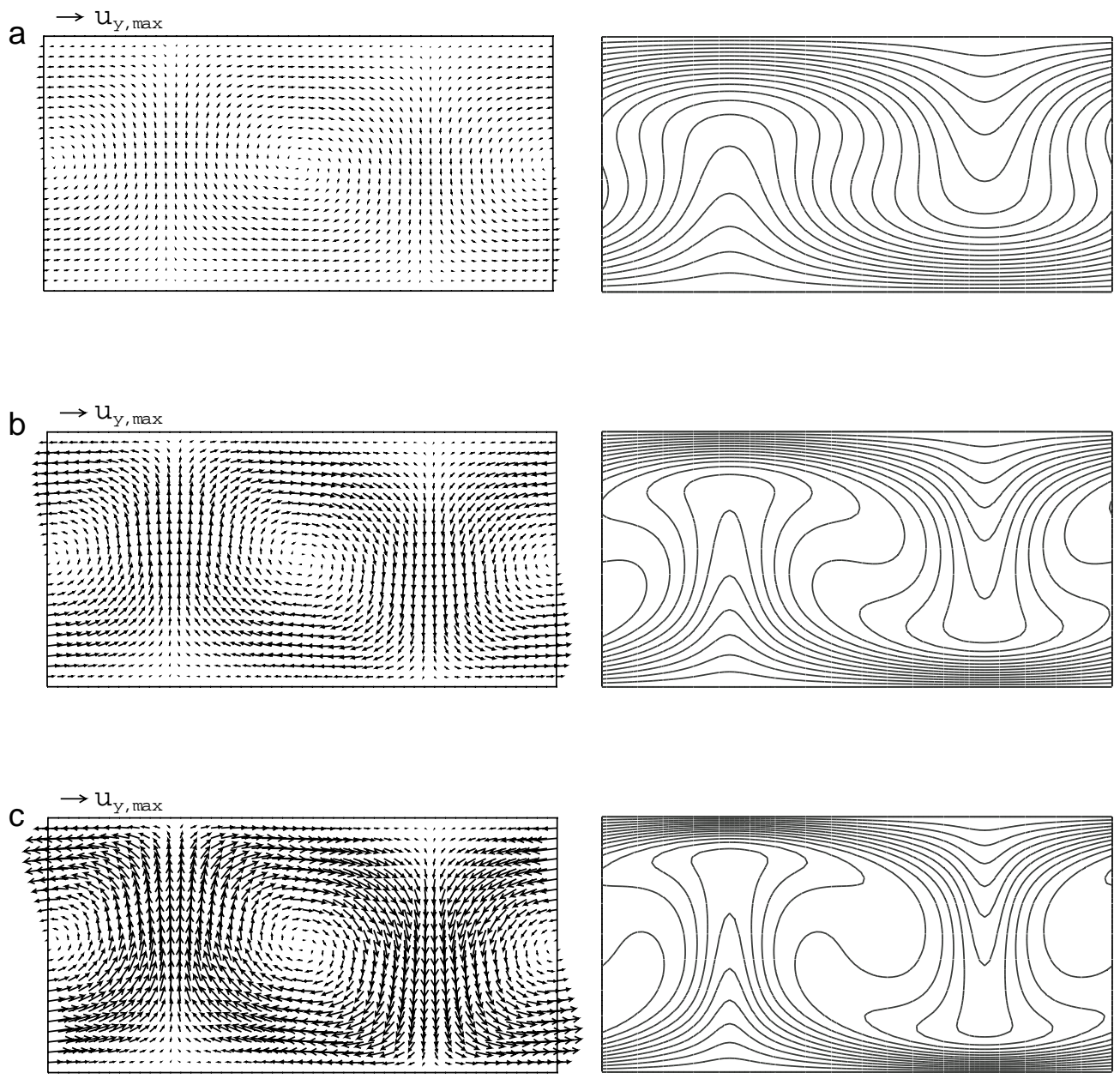

FIG. 4. Calculated velocity vectors (left) and normalized temperature $\left(T-T_{\mathrm{U}}\right) /\left(T_{\mathrm{L}}-T_{\mathrm{U}}\right)$ where $T_{\mathrm{L}}$ and $T_{\mathrm{U}}$ are the temperatures on the lower and upper walls, respectively (right), in final steady-states at different Rayleigh numbers. (a) $\mathrm{Ra}=5,000$, (b) $\mathrm{Ra}=20,000$, and (c) $\mathrm{Ra}=50,000$. $u_{y, \max }$ is the maximum velocity in the $y$-direction at $\mathrm{Ra}=50,000$ and temperature contour interval is 0.05 .

\section{R A F T February 15, 2002, 1:27pm D R A F T}




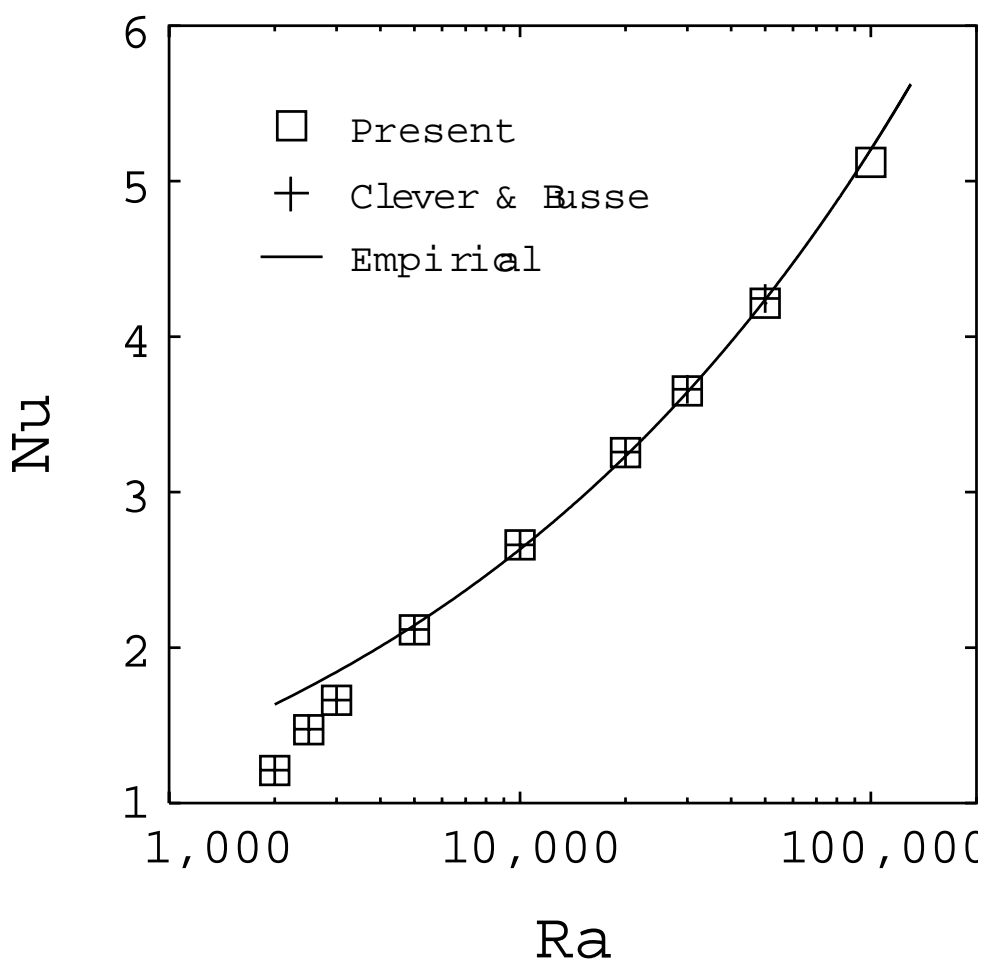

FIG. 5. Calculated relationship between the Nusselt number and the Rayleigh number. The results by Clever and Busse [26] and the empirical formula $\mathrm{Nu}=1.56\left(\mathrm{Ra} / \mathrm{Ra}_{\mathrm{c}}\right)^{0.296}[16]$ where $\mathrm{Ra}_{\mathrm{c}}$ is the critical Rayleigh number are also shown for comparison.

D R A F T February 15, 2002, 1:27pm D R A F T 